http://doi.org/10.48195/sepe2021-129

\title{
FISIOTERAPIA OCULAR: UMA ÁREA AINDA POUCO EXPLORADA
}

\section{Camila Wayss Cocco2; Carlos Eduardo Bueno'; Juliana Saibt Martins 3 ; Pietra Alves $^{4}$; Samara Tondo ${ }^{5}$; Sheila Spohr Nedel ${ }^{6}$;Nadiesca Filippin ${ }^{7}$;}

\section{RESUMO}

A Fisioterapia Ocular é importante no tratamento de alterações oculares onde sua finalidade é mudar os mecanismos neurofisiológicos do comando vergênero por meio da estimulação visual repetida, contribuindo para uma melhor qualidade de vida e conforto visual. $O$ objetivo deste trabalho revisar a literatura sobre a Fisioterapia Ocular, uma área ainda pouco explorada e conhecida. Este trabalho trata-se de uma revisão na literatura sobre a Fisioterapia Ocular, onde foram descritos aspectos relacionados à anatomia e fisiologia ocular, relação entre controle postural e sistema visual, disfunções posturais associadas a disfunções oculomotoras e visuais e como a fisioterapia atua frente a essas condições. Segundo a literatura o sistema oculomotor e visual tem grande influência sobre o sistema musculoesquelético, contribuindo para o controle postural, equilíbrio, entre outros. E, portanto, demonstrando a importância de trabalhar os estímulos visuais e a musculatura ocular para recuperar as funções motoras, funcionalidade e a independência para realizar atividades.

\footnotetext{
${ }^{1}$ Carlos Eduardo Bueno Peixoto Belo - Acadêmico do curso de Fisioterapia da Universidade Franciscana (UFN). carlos.eduardo.bueno22@gmail.com

${ }^{2}$ Camila Wayss Cocco - Acadêmico do curso de Fisioterapia da Universidade Franciscana (UFN). camilawayss@hotmail.com

3 Juliana Saibt Martins - Docente do Curso de Fisioterapia e do Mestrado em Ciências da Saúde e da Vida da Universidade Franciscana (UNF). jsaibt@prof.ufn.edu.br

${ }_{4}^{4}$ Pietra da Rosa Alves - Acadêmico do curso de Fisioterapia da Universidade Franciscana (UFN). pietradarosa.pr@gmail.com

${ }^{5}$ Samara Tondo - Acadêmico do curso de Fisioterapia da Universidade Franciscana (UFN). samarastondo@gmail.com

${ }^{6}$ Nadiesca Filippin - Docente do curso de Fisioterapia e do Mestrado Profissional em Saúde Materno Infantil da Universidade Franciscana (UFN).nadifilippin@prof.ufn.edu.br

${ }^{7}$ Sheila Spohr Nedel - Docente do Curso de Fisioterapia da Universidade Franciscana (UFN).

sheila.nedel@prof.ufn.edu.br
} 
Palavras-chave: Controle postural, Défict oculomotor; Fisioterapia; Visão.

Eixo Temático: Atenção integral e promoção a saúde.

\section{INTRODUÇÃO}

A Fisioterapia Ocular, objetiva mudar os mecanismos neurofisiológicos do comando vergênico, através de repetições de um estímulo visual, com a intenção de estimular o cérebro por inteiro, com foco nos centros que são responsáveis pelas tarefas visuais (FONTINELE et al., 2019; KMETZI, 2019).

Nossos olhos são formados por camadas, a esclera que é a primeira camada, que reveste quase todo olho, menos a córnea, depois a camada coróide, que produz a melanina que atua na filtração dos raios solares. Na última camada temos a retina que é uma película fina formada por células competentes em capturar a luz e estímulos que são transmitidos ao nervo óptico (TORTORA; BRYAN, 2016).

$\mathrm{Na}$ anatomia ocular também temos músculos que são extremamente importantes para o funcionamento adequado dos olhos, como reto medial, reto lateral, reto inferior, reto superior, oblíquo inferior e oblíquo superior (MAIA, 2018).

Deste modo, conseguimos entender toda a formação e funcionamento visual e considerar que existem outras funções do nosso corpo que podem estar diretamente relacionadas com a função e movimento ocular, como por exemplo, o controle postural.

Quando temos alguma alteração visual, podemos consequentemente ter também alterações posturais, pois o sentido da visão tem função fundamental na orientação e estabilidade do nosso corpo (ROLL; VEBEL, 1989).

Considerando esses aspectos, a fisioterapia é de extrema importancia para que aconteça uma melhora significativa do paciente em um contexto geral, não atuando apenas da patologia, mas também nas consequências que ela pode causar em outros sistemas do corpo humano.

Portanto, este estudo teve por objetivo revisar a literatura sobre a Fisioterapia 
Ocular, uma área ainda pouco explorada e conhecida.

\section{METODOLOGIA}

O presente artigo trata-se de uma revisão de literatura em que foram utilizadas as bases de dados online PubMed, SciELO, PEDro e LILACS. A pesquisa foi desenvolvida a partir da necessidade de um conhecimento maior sobre a área da fisioterapia ocular, tendo em vista os efeitos que a visão exerce sobre o controle postural. Inicialmente na busca de dados foram analisados títulos e resumos referente ao tema abordado, na qual utilizou-se as palavras chave "controle postural", "défict oculomotor", "fisioterapia" e "visão".

\section{ANATOMIA E FISIOLOGIA OCULAR}

A visão, o ato de ver, é extremamente importante para a sobrevivência humana. Mais da metade dos receptores sensitivos no corpo humano estão localizados nos olhos e uma grande parte do córtex cerebral é dedicada ao processamento da informação visual de acordo com Tortora e Bryan (2016).

Os olhos são compostos por algumas estruturas básicas, começando pelos fotorreceptores, tais como: a esclera que é a primeira camada que cobre todo o bulbo do olho, exceto a córnea, dando formato ao bulbo e o tornando mais rígido, protege suas partes internas e age como um local de fixação para os músculos extrínsecos do bulbo do olho (TORTORA; BRYAN, 2016). Em seguida vem a segunda camada chamada de coroide a qual contém melanócitos que produzem o pigmento melanina atuando na absorção de raios solares dispersos, evitando a reflexão e a dispersão de luz dentro do bulbo do olho (MAIA, 2018). E a terceira camada é composta pela retina sendo o início da via visual e tendo morfologia neurossensorial que contém células altamente especializadas em capturar a luz externa e processar os estímulos resultantes transmitindo-os ao nervo óptico (MAIA, 2018). Na camada da retina ainda há a fóvea que é uma depressão no centro da mácula, estruturas essas responsáveis pela resolução da imagem que enxergamos (TORTORA; BRYAN, 2016). 
Além dos componentes fotorreceptores também há a parte de refração da luz composta pela córnea tendo como principal função a refração óptica, sendo que para a excelência da função deve haver as curvaturas, transparência e estabilidade do filme lacrimal. E possui o cristalino que com a convergência correta faz com que a imagem formada na retina fique nítida e bem definida (CRISPIM et al., 2019).

Para o controle da entrada de luz há a íris que fornece a cor aos olhos, formada por melanócitos e fibras musculares lisas, tem como principal função a regulação da quantidade de luz que entra no bulbo do olho através da pupila, abertura no centro da íris, que na presença de muita luz se constringe e quando há pouca ou ausência de luz, se dilata (MAIA, 2018).

Por fim, De Lucía et al. (2018) citam os músculos extraoculares que são responsáveis pela movimentação e fixação dos olhos, sendo eles: reto medial (realiza adução), reto lateral (realiza abdução), reto inferior (realiza abaixamento, adução e de exciclodução), reto superior (realiza elevação, adução e de inciclodução), oblíquo inferior (realiza exciclodução, elevação e de abdução) e oblíquo superior (realiza e inciclodução, abaixamento e de abdução). Esses movimentos...

Dessa forma, pode-se observar a complexidade da visão e do movimento ocular e presumir o quanto essas funções podem influenciar na execução dos movimentos corporais.

\section{INFLUÊNCIA DA VISÃO NO CONTROLE POSTURAL}

Segundo Roll; Vebel e Roll (1989), a maioria das atividades realizadas por nós seres humanos tem como fator fundamental a compreensão visual de um alvo, sendo que este tem um importante papel na orientação do corpo e dos membros. Isso é possível graças à grande mobilidade do globo ocular, que permite que os olhos estejam sempre observando o ambiente à sua volta e assim informando o restante do corpo, o preparando para futuras ações e para adotar posturas adequadas para o momento ou para algum futuro movimento corporal.

O estímulo através da vibração mecânica de diferentes combinações de áreas 
do globo ocular produz deslocamentos corporais, para frente, para trás ou para os lados, dependendo do par de músculos oculares estimulados (ROLL; VEBEL; ROLL, 1989). Isso indica que há uma grande ligação entre os movimentos dos olhos e a organização espacial e a postura corporal, sendo que a musculatura dos olhos e, portanto, o movimento dos olhos, e não apenas as retinas, contribuem para a regulação e controle corporal (ROLL; VEBEL; ROLL, 1989; CUNHA, 1987; GAGEY, 1987; MARRUCHI; GAGEY, 1987).

Alguns estudos estabelecem uma relação entre o sistema visual e os músculos do trapézio e da região da cervical e do pescoço, em que os pacientes com dores nessas áreas relataram também sintomas de distúrbios visuais (TRELEAVEN; TAKASAKI, 2014; TRELEAVEN, 2017). O que vai ao encontro do que Pastor (2012) constatou, de que para se ter a localização espacial é necessário o sistema oculomotor e os músculos responsáveis por movimentar a cabeça íntegros, o que torna possível compreender a relação entre esses dois sistemas e as possíveis alterações posicionais e dinâmicas que podem ocorrer caso um deles sofra alguma alteração.

Zhang et al. (2016) observaram que crianças com alcance de fusão horizontal reduzido para convergência ou divergência apresentavam uma postura anômala da cabeça, em que pode-se perceber que, devido ao estreitamento da visão binocular, as crianças acabavam por fazer uma rotação da cabeça como forma de compensação. Outro mecanismo de adaptação que pode ocorrer devido aos distúrbios oculomotores são a ambliopia, supressão, correspondência sensorial anômala, fixação excêntrica e a posição viciosa da cabeça (PASTOR, 2012).

O equilíbrio também tem relação com os estímulos visuais o que pode se observar em estudos que apontam que intervenções que trabalham os movimentos dos olhos, como os exercícios de movimentos dos olhos e equilíbrio, acabam sendo mais eficazes em melhorar o equilíbrio e a reduzir as tonturas e fadigas (HERBERT et al., 2018). Roll; Vedel e Roll (1989) sugerem que existe uma forte ligação entre os olhos e o suporte plantar por meio das informações proprioceptivas musculares.

Assim, a marcha também sofre alterações de acordo com os sentidos, bem 
como outros movimentos corporais podem ser influenciados por estímulos visuais (HOLLANDS et al., 2015). Segundo Young e Hollands (2010; 2012), é comum encontrar em grupos com alto risco de quedas alguma alteração comportamental visual durante a marcha. Corroborando este pensamento, um estudo feito por Hollands et al. (2015), com pacientes pós acidente vascular cerebral (AVC), indicou que alterações na marcha podem apresentar melhorias, como na velocidade da marcha, simetria do comprimento do passo e o tempo de apoio único, graças a estímulos/dicas visuais durante o treino da marcha.

Além disso, estudos feitos por Flachenecker et al. (2002) e Feys et al. (2008) apresentaram que pacientes com instabilidade ocular e esclerose múltipla (EM) acabam indicando aumento de tremores de intenção durante as atividades motoras que requerem a coordenação simultânea dos olhos e das mãos, o que indica a forte relação entre capacidade visual e a motora.

O que se pode observar com isso é que disfunções oculomotoras e visuais podem comprometer as funções motoras e a funcionalidade contribuindo, assim, para a perda de independência para realizar as atividades de vida diária. Com isso, um acompanhamento multiprofissional torna-se necessário, sendo o fisioterapeuta um membro importante dessa equipe.

\section{FISIOTERAPIA OCULAR}

Os fisioterapeutas são profissionais de grande importância quando se trata do tratamento de alterações oculomotoras, pois previnem as possíveis sequelas, melhoram o conforto visual e melhoram a qualidade de vida das pessoas (FONTINELE et al., 2019).

O objetivo da terapia visual ativa não é justamente desenvolver a força muscular, mas mudar os mecanismos neurofisiológicos por meio da estimulação visual repetida. A ideia é estimular todo o cérebro, em especial os centros responsáveis pelas tarefas visuais, em que a resposta muscular é apenas uma parte da visão binocular (CALOROSO; ROUSE, 1993; FONTINELE et al., 2019; KMETZI; 
TAGLIETTI, 2019).

A fisioterapia tem apresentado resultados significativos na recuperação do alinhamento ocular e da visão binocular, por meio da realização de treinamentos dos movimentos dos músculos extrínsecos dos olhos (JANG et al., 2017). O treinamento tem apresentado diversos benefícios ao paciente como desenvolvimento do conforto visual, qualidade de vida e prevenção da evolução das alterações que tenham necessidade cirúrgica ( SCHEIMAN; GWIAZDA; LI, 2011).

$\mathrm{Na}$ literatura são encontrados alguns estudos sobre os efeitos da fisioterapia ocular em diferentes condições de saúde, em amostras de diferentes idades. Bayram; Karagöz e Algun (2020) realizaram um estudo sobre o efeito dos exercícios de Bates, convergência e oculomotores atuando na hipermetropia da população em idade escolar. O estudo durou um período de 6 semanas, em que os participantes escolhidos tinham idades entre 7 e 17 anos, com diagnóstico de hipermetropia. Foram incluídos 60 pacientes e esses foram divididos em 3 grupos, em que cada grupo realizou um exercício dos três apresentados. Os autores concluíram que os exercícios oculomotores e de convergência ajudaram na acuidade visual e na qualidade de vida.

Segundo estudo de Wyk e colaboradores (2014), o treinamento de movimentos dos olhos com exercícios de varredura visual apresentam um efeito positivo significativo na percepção visual em pacientes com Negligência Espacial Unilateral (NEU) após AVC. Foram analisados 24 indivíduos entre 19 e 74 anos, divididos em dois grupos de 12 pessoas, sendo um grupo controle e o outro experimental, em que o grupo experimental recebeu treinamento de movimento ocular sacádico com varredura visual por um período de 4 semanas.

Já no estudo de Gandolfi et al. (2016), os autores compararam os resultados após o treinamento de orientação postural com estímulos visuais e somatossensoriais versus fisioterapia convencional em pacientes com Síndrome de Pusher (SP) após AVC, e concluíram que esse protocolo de fisioterapia pode reduzir a severidade da $\mathrm{SP}$, como também, melhorar o controle postural do paciente com este comprometimento. 
Armstrong (2018) relata que pacientes pós TCE apresentam defeitos na visão como a percepção visual, movimentos dos olhos incluindo vergência, acinetopsia e acuidade visual, em que tratamentos como o treinamento oculomotor, reabilitação visual incluindo a leitura, a prescrição de óculos com diversas tonalidades e combinações de prisma foram benéficos para tratar essas disfunções.

Nesse contexto, pode-se observar a infinidade de disfunções, de diferentes origens e causas, passíveis de serem tratadas pela fisioterapia ocular, em pessoas de diferentes faixas etárias. Além disso, torna-se relevante destacar a associação dos diferentes sistemas do corpo e seu funcionamento integrado, que permitem a execução correta dos movimentos, manutenção da estabilidade e orientação corporais fundamentais para a conservação da funcionalidade dos indivíduos.

\section{CONCLUSÃO}

Diante do exposto pode-se destacar o quanto a anatomia e fisiologia ocular é complexa e o quanto o sistema visual e oculomotor podem interferir em outros sistemas de nosso corpo, como por exemplo, o sistema de controle postural. Devido a essa interação, as disfunções observadas em qualquer parte do sistema visual e/ou oculomotor podem comprometer o sistema de ajuste postural e, com isso, provocar déficits funcionais. Dessa forma, a fisioterapia ocular, uma área ainda pouco estudada, aparece com destaque, pois através de diferentes intervenções pode produzir efeitos benéficos sobre a saúde funcional do indivíduo com algum tipo de disfunção visual e/ou oculomotora.

\section{REFERÊNCIAS}

AIRES, Margarida de Mello. Fisiologia. 4. ed. Rio de Janeiro: Guanabara Koogan, 2012.

ARAS BAYRAM, Gülay; KUTLUTÜRK KARAGÖZ, Işı; ALGUN, Zeliha Candan. 
Investigação do efeito de diferentes exercícios para os olhos na hipermetropia em crianças em idade escolar: um estudo duplo-cego randomizado, 2020.

ARMSTRONG, Richard A. Problemas visuais associados a lesão cerebral traumática. Clinical and Experimental Optometry, v. 101, n. 6, pág. 716-726, 2018.

CALOROSO EE, ROUSE MW. Clinical management of strabismus, Boston, 1993, Butterworth-Heinemann, pg. 32-39, 1999.

CUNHA, H. M. M. da. Le syndrome de deficience posturale (SDP). Agressologie, França, v. 28, p. 941 - 943, out. 1987.

DE LUCÍA et al. UNIVERSIDAD COMPLUTENSE DE MADRID FACULTAD DE MEDICINA DEPARTAMENTO DE OFTALMOLOGÍA Y OTORRINOLARINGOLOGÍA TESIS DOCTORAL Estudio de los músculos extraoculares mediante tomografía de coherencia óptica de segmento anterior MEMORIA PARA OPTAR AL GRADO DE DO. [s. I.], 2018. Disponível em: http://eprints.ucm.es/47040/1/T39784.pdf

FEYS, P. et al. Unsteady gaze fixation enhances the severity of MS intention tremor. Neurology, Bélgica, v. 70, p. 106-113, jan. 2008.

FLACHENECKER, P. et al. Fatigue in multiple sclerosis: a comparison of different rating scales and correlation to clinical parameters. Multiple Sclerosis Journal, Alemanha, v. 8, n. 6, p. 523-526, 2002.

Fisiologia / Linda S. Costanzo; revisão técnica Carlos Alberto Mourão Júnior. - 6. ed. Rio de Janeiro : Guanabara Koogan, 2015.

FONTINELE, Amanda Gabriela Mendes et al. Reabilitação oculomotora: novos desafios na prática clínica fisioterapêutica. CIPEEX, v. 2, p. 1708-1708, 2018.

Foxe JJ, Simpson GV, Ahlfors SP. Parieto-occipital approximately $10 \mathrm{~Hz}$ activity reflects anticipatory state of visual attention mechanisms. Neuroreport. 1998 Dec 1;9(17):3929-33. doi: 10.1097/00001756-199812010-00030. PMID: 9875731.

GAGEY, P. M. L'oculomotricité comme endo-entrée du système postural. Agressologie, França, v. 28, p. 899-903, out. 1987.

GANDOLFI, M; GEROIN, C; FERRARI, F; LA MARCHINA, E; VARALTA, V; FONTE, $\mathrm{C}$; et al. Procedimentos de reabilitação no manejo dos déficits de orientação postural em pacientes com comportamento de empurrador pós-AVC: um estudo piloto. Minerva Med, dezembro de 2016; 107 (6): 353-62.

HERBERT, J. R. et al. Efficacy of Balance and Eye-Movement Exercises for Persons With Multiple Sclerosis (BEEMS). Neurology. Aurora, v. 90, p. 797-807, jan. 2018. 
HOLLANDS, K. L. et al. Feasibility and preliminary efficacy of visual cue training to improve adaptability of walking after stroke: multi-centre, single-blind randomised control pilot trial. PLoS one, Reino Unido, v. 10, p. 1-17, out. 2015.

JANG, Jung Un et al. Eficácia da terapia visual em escolares com insuficiência de convergência sintomática. Journal of ofthalmic \& vision research , v. 12, n. 2, pág. $187,2017$.

KMETZI, R., \& i,TAGLIETTI M. EFETIVIDADE DOS EXERCÍCIOS DOMICILIARES NA INSUFICIÊNCIA DE CONVERGÊNCIA. FAG JOURNAL OF HEALTH (FJH), 1(2),

88-96., 2019.

MAIA, Núbia Cristina de Freitas. Fundamentos básicos da oftalmologia e suas aplicações. Palmas: Eduft, 2018. 122 p.

MARUCCHI, C.; GAGEY, P. M. Cécité posturale. Agressologie, França, v. 28, p. 947948, out. 1987.

Oftalmologia para a graduação [recurso eletrônico]/ Organizadores: João Crispim Moraes Lima Ribeiro et al. - Fortaleza: EdUnichristus, 2019. Disponível em: www.unichristus.edu.br

PASTOR, I. P. Terapia manual en el sistema oculomotor. Espanha: Elsevier, 2012.

Princípios de anatomia e fisiologia / Gerard J. Tortora, Bryan Derrickson; tradução Ana Cavalcanti C. Botelho... [et al.]. - 14. ed. - Rio de Janeiro: Guanabara Koogan, 2016.

Pollock A, Hazelton C, Rowe FJ, Jonuscheit S, Kernohan A, Angilley J, Henderson CA, Langhorne P, Campbell P. Interventions for visual field defects in people with stroke. Cochrane Database of Systematic Reviews 2019, Issue 5. Art. No.: CD008388. DOI: 10.1002/14651858.CD008388.pub3.

ROLL, J. P.; VEDEL, J.P; ROLL, R. Eye, head and skeletal muscle spindle feedback in the elaboration of body references. Prog Brain Res., França, v. 80, p. 113-23, 1989.

SANTOS, S. M. T.; NISHIMOTO, M. G.; PIERUCCI, A. et al. Equilíbrio em Pacientes com Traumatismos Encefálicos que Praticam Natação e Realidade Virtual. Rev Neurocienc., v.21, n.1, p.89-93, 2013.

SCHEIMAN, Mitchell; GWIAZDA, Jane; LI, Tianjing. Intervenções não cirúrgicas para insuficiência de convergência. Cochrane Database of Systematic Reviews , n. 3, 2011. 
TRELEAVEN, J. Dizziness, unsteadiness, visual disturbances, and sensorimotor control in traumatic neck pain. J. Orthop. Sport Phys. Ther. Australia, v. 47, p. 492502, jun. 2017.

TRELEAVEN, J.; TAKASAKI, $\mathrm{H}$. Characteristics of visual disturbances reported by subjects with neck pain. Man. Ther. Australia, v. 19, p. 203-207, jun. 2014.

VAN WYK, Andoret; EKSTEEN, Carina A .; RHEEDER, Paul. O efeito dos exercícios de varredura visual integrados à fisioterapia em pacientes com negligência espacial unilateral pós-AVC: um ensaio de controle randomizado de pares combinados. Neurorreabilitação e reparo neural , v. 28, n. 9, pág. 856-873, 2014.

YOUNG, W. R.; HOLLANDS, M. A. Can telling older adults where to look reduce falls? Evidence for a causal link between inappropriate visual sampling and suboptimal

stepping performance. Exp Brain Res., Birmingham, v. 204, p. 103-113, jul. 2010.

YOUNG, W. R.; HOLLANDS, M. A. Newly acquired fear of falling leads to altered eye movement patterns and reduced stepping safety: a case study. PLoS One, Liverpool, v. 7, p. 1-7, nov. 2012.

ZHANG, D. et al. Binocular vision and abnormal head posture in children when watching television. Int. J. Ophthalmol. China, v. 9, p. 746-749, maio 2016. 